A N N A L E S Annales de Bretagne et des Pays de l'Ouest

Anjou. Maine. Poitou-Charente. Touraine

$115-4 \mid 2008$

Varia

\title{
La mort et le deuil de la pêche langoustière bretonne en Mauritanie
}

Françoise Pencalet-Kerivel

\section{OpenEdition}

Édition électronique

URL : http://journals.openedition.org/abpo/228

DOI : $10.4000 / a b p o .228$

ISBN : 978-2-7535-1512-3

ISSN : 2108-6443

Éditeur

Presses universitaires de Rennes

Édition imprimée

Date de publication : 31 décembre 2008

Pagination : 173-200

ISBN : 978-2-7535-0808-8

ISSN : 0399-0826

Référence électronique

Françoise Pencalet-Kerivel, «La mort et le deuil de la pêche langoustière bretonne en Mauritanie », Annales de Bretagne et des Pays de l'Ouest [En ligne], 115-4 | 2008, mis en ligne le 31 décembre 2010 consulté le 19 avril 2019. URL : http://journals.openedition.org/abpo/228 ; DOI : 10.4000/abpo.228

(c) Presses universitaires de Rennes 


\title{
La mort et le deuil de la pêche langoustière bretonne en Mauritanie
}

\author{
Françoise Pencalet-KeRIVEL \\ docteur en histoire, chercheur associé au CERHIO \\ professeur d'histoire-géographie au lycée Brizeux (Quimper)
}

De 1955 à 1990, la pêche à la langouste rose a dynamisé l'économie portuaire de deux ports bretons, Douarnenez et Camaret. Lors de sa naissance en 1955, elle se pratiquait dans les eaux libres au large des côtes de l'Afrique Occidentale Française (AOF). En 1960, la décolonisation de la Mauritanie et surtout l'édification du droit de la mer modifient les règles du jeu : le nouvel État indépendant étend progressivement ses eaux territoriales ${ }^{1}$ jusqu'à atteindre une zone économique de pêche ${ }^{2}$ de 200 milles nautiques en 1978. Avec l'extension des eaux territoriales, la pêche à la langouste rose s'effectue dans des eaux sous juridiction mauritanienne. Pour obtenir le droit d'y pêcher, la France négocie avec la République Islamique de Mauritanie (RIM). Les exigences mauritaniennes s'accroissent à partir de 1973 : elles imposent à la France l'embarquement de marins mauritaniens et des licences de pêche dont le coût devient prohibitif. Acculés, les pêcheurs langoustiers bretons se tournent vers la CEE. Depuis les conventions de Lomé (en 1975 et 1979), la CEE favorise " dans le cadre des coopérations industrielles, financières et techniques, dans les États Afrique-Caraïbes-Pacifique (ACP) qui en manifestent l'intérêt, le développement de la pêche et des industries afférentes ${ }^{3}$, elle négocie donc avec de nombreux États africains ${ }^{4}$. En 1983, les pouvoirs de la CEE dans le domaine des pêches se renforcent ${ }^{5}$ : lorsque les accords

1. Zone parallèle à la côte qui est considérée comme partie du territoire national et dans laquelle l'État côtier exerce pleinement sa souveraineté.

2. Zone au-delà de la ligne de base, dans laquelle les navires de l'État côtier peuvent conserver l'exclusivité du droit de pêche.

3. Archives de l'Union des Armateurs de la Pêche Française (UAPF), dossier Mauritanie, extrait du JO des Communautés européennes, $n^{\circ}$ L25/122 du 30 janvier 1976. Protocole $\mathrm{n}^{\circ} 7$, annexe.

4. CASADA RAIGON, Rafael, L'Europe et la mer : pêche navigation et environnement marin, Bruxelles, Bruylant, Édition de l'Université de Bruxelles, 2005.

5. SoRAIN, Dominique, "La modernisation de la pêche et son adaptation à l'Europe bleue ", Regards sur l'actualité, nº 228, février 1997, p. 19. 
bilatéraux expirent, la communauté se substitue aux pays membres dans leurs relations avec les pays en développement. Ainsi, l'accord négocié entre la CEE et la République Islamique de Mauritanie, en 1987, s'inscrit dans le vaste réseau d'accords de pêche de la CEE avec divers pays ACP. Après avoir été porteur d'espoir pour les langoustiers bretons, l'accord provoque la mort de la pêche langoustière. Dans les années quatre-vingt, l'activité langoustière représentait $15 \%$ du chiffre d'affaires du port de Douarnenez et $75 \%$ de celui de Camaret. Comment, dès lors, les deux ports bretons acceptent la mort de l'activité et tournent la page langoustière? Comment acceptent-ils la transformation de l'outil de travail, du bateau appelé " mauritanien ${ }^{6}$ ", en objet du patrimoine?

\section{L'accord communautaire : entre espoir et désillusion}

\section{L'accord communautaire de 1987}

" Compte tenu des compétences de la CEE en matière de politique extérieure de la pêche, notamment en ce qui concerne les rapports des états membres avec les pays tiers, il n'est plus possible pour la France de négocier un nouvel accord bilatéral avec la Mauritanie ${ }^{7}$."

Entre cette affirmation de janvier 1979 et la réalisation des accords en 1987, huit années de négociations sont nécessaires pour aboutir au texte final. Ce difficile aboutissement s'explique par les problèmes politiques de la Mauritanie en 1979 et par les divergences de vues entre les deux parties (divergences en ce qui concerne la création d'une société mixte souhaitée par la Mauritanie, les prix des licences de pêche ${ }^{8}$ et de la compensation communautaire et l'embarquement des marins mauritaniens ${ }^{9}$ ). Après les premiers échecs, la convention de Lomé III en 1984 accélère le processus en imposant aux États ACP l'obligation nouvelle de négocier des accords de pêche avec la Communauté lorsque celle-ci le demande ${ }^{10}$. En octobre 1984,

6. " mauritanien " : navire langoustier pêchant sur les côtes de la Mauritanie. Écrit avec une majuscule, "Mauritanien " désigne le marin pêcheur de Douarnenez ou de Camaret pêchant la langouste sur les côtes de Mauritanie.

7. Archives de la société brestoise FL Kuhn. Carton Mauritanie 1. Document confidentiel du ministère des transports, direction générale de la Marine Marchande, direction des pêches maritimes. Compte rendu de la réunion du 11/01/1979 concernant la pêche langoustière en Mauritanie. Document rédigé par B. Labrousse, sous-directeur des pêches maritimes.

8. Licences de pêche : accord autorisant les flottes étrangères à pêcher dans les eaux territoriales ou la ZEE d'un pays. Les licences supposent généralement le versement d'une redevance en contrepartie des droits d'accès à la ressource, elles indiquent la taille de la flottille autorisée, le volume maximal des prises et les espèces pouvant être capturées.

9. Archives de la société brestoise FL Kuhn. Mauritanie 2. ministère de la Mer, Direction des pêches maritimes et des cultures marines, Paris, le 22 mars 1983.

10. Archives de la société brestoise FL Kuhn. Mauritanie 2. Fiche du secrétariat d'État à la mer sur les activités des pêcheurs français en Mauritanie et les négociations d'accords de pêche en 1986. 
des consultations informelles en vue d'un accord de pêche se déroulent à Bruxelles ${ }^{11}$, elles aboutissent au protocole de 1987.

Le protocole d'accord paraphé le 14 mai $1987^{12}$ entre en vigueur le $1^{\mathrm{er}}$ juillet de cette même année. Il est conclu pour une période de trois ans. Cette durée de l'accord permet aux armateurs français d'envisager plus sereinement l'avenir de l'activité. Comme dans le cadre des accords bilatéraux, le droit de pêcher s'obtient contre le paiement d'une licence délivrée pour un bateau donné et pour une période de douze mois. Les armateurs payent une redevance annuelle de 121 écus par tonneaux de jauge brute (tjb) ${ }^{13}$, la Communauté la complète en versant une compensation financière à la Mauritanie. De ce fait, dans le cadre communautaire, le coût des licences est moins élevé pour les armateurs, ce qui représente une économie de l'ordre de $300000 \mathrm{~F}$ par navire ${ }^{14}$. L'accord impose également un relevé et une déclaration mensuelle des captures auprès du Centre National de Recherche Océanographique et de Pêche de Mauritanie (CNROP) ${ }^{15}$. Chaque navire doit embarquer des marins pêcheurs mauritaniens à hauteur de $35 \%$ de l'équipage. L'accord autorise la pêche des langoustiers pour un tonnage global de 3500 tjb par mois en moyenne annuelle.

Jusqu'en 1986, les autorités mauritaniennes autorisaient l'accès à leurs eaux à une flottille langoustière française de 2561 tjb correspondant à dix langoustiers bretons : Rio del Oro, Iroise, Joliot-Curie, Claire-Jeanne et NotreDame-De-Rocamadour de Douarnenez, et, Saint-Rioc, Equateur, Armorique, Castel Dinn et Portzic de Camaret. En 1984, le Portugal a signé avec la RIM un accord de pêche triennal pour des chalutiers et des langoustiers ${ }^{16}$. Ainsi, avant l'accord communautaire, deux à quatre langoustiers portugais pêchaient dans les eaux mauritaniennes ${ }^{17}$. Au moment de la négociation des accords CEE-Mauritanie, le Portugal privilégiait la pêche démersale ${ }^{18}$ aux sparidés qu'ils considéraient comme plus rentable que celle à la langouste. Mais en janvier 1987, le Portugal perd ses droits pour la pêche démersale

11. Archives de la CEE. Direction générale de la pêche. XIV/A/2. Note de dossier le 21 octobre 1984.

12. Archives FLK. Camaret 6 . Texte publié au journal officiel des Communautés européennes le 31 décembre 1987, $\mathrm{JO} \mathrm{n}^{\circ} \mathrm{L} 388 / 1$.

13. La jauge brute est une mesure de la capacité de transport d'un navire, elle s'exprime en tonneaux de jauge brute (tjb).

14. Archives de la société brestoise FL Kuhn. Mauritanie 2. Information extraite du dossier « situation de la pêche langoustière en Mauritanie ", fin 1988.

15. Centre National de Recherches Océanographiques et des Pêches établi dans la baie du Lévrier, au sud de la ville de Nouadhibou.

16. Archives de la société brestoise FL Kuhn. Carton armoire LK. Mauritanie 1 : Procèsverbal des discussions mauritano-portugaises du 3 au 6 janvier 1984.

17. Archives CEE. Direction générale des pêches. XIV/A/2. Compte rendu du groupe externe du 15 juin 1987, Mauritanie.

18. Poissons démersaux : les poissons démersaux ou poissons de fond vivent au fond de la mer et ont un habitat constant. Par l'expression " espèce démersales " on désigne parfois aussi les céphalopodes (poulpes, seiches et calmars) qui vivent également au fond de la mer. Les langoustes vivent au fond de la mer. 
car la Mauritanie décide de la réserver à ses pêcheurs nationaux. En compensation le Portugal demande à la Commission Européenne des licences pour la pêche à la langouste ${ }^{19}$. Comme le Portugal est le seul pays de la CEE à avoir un accord gouvernemental en cours avec la RIM, la CEE, en vertu de l'article 354 de l'Acte d'adhésion ${ }^{20}$, doit rechercher des solutions alternatives pour ce pays. Pour cette raison, la CEE répartit entre Français et Portugais les 3500 tjb autorisés pour la pêche à la langouste : 2627 tjb pour les Français et 873 tjb pour le Portugal. Le protocole précise que les langoustiers sont des caseyeurs ${ }^{21}$, mais, à titre dérogatoire pendant la première année d'application du protocole, les navires de la Communauté peuvent utiliser des filets maillants pour un tonnage global ne dépassant pas 873 tjb (tonnage correspondant aux navires portugais).

\section{L'espoir mauritanien et communautaire}

Avec l'accord de 1987, les Mauritaniens comme les Français envisagent le développement de la pêche langoustière. L'espoir mauritanien de développer sa propre pêcherie langoustière se concrétise. En avril 1987, le gouvernement mauritanien précise que « la nouvelle stratégie encourage la constitution d'un armement national qui a la priorité de l'exploitation des ressources en crustacés (crevettes et langoustes), afin d'accroître les retombées locales de cette exploitation ${ }^{22}$ ". Un rapport de la Food and Agriculture Organization (FAO) ${ }^{23}$ de l'organisation des Nations Unies (ONU) prévoit la création d'un armement langoustier mauritanien composé d'une quinzaine de navires en remplacement des vieux langoustiers bretons et en utilisant les services de cadres pêcheurs bretons. L'aide des Bretons est essentielle car " en Mauritanie pour l'instant, il n'y a ni armateur, ni marins pêcheurs ayant le savoir-faire particulier à cette pêche pour les capturer, les conserver vivantes et pour les commercialiser sur le marché en Europe, marché certes très porteur mais aussi très fermé ${ }^{24}$ ». Les auteurs du rapport envisagent le développement de l'activité langoustière mauritanienne d'autant

19. Archives CEE. Direction générale des pêches. Note de dossier du 15 juin 1987 sur la demande portugaise de licence pour la pêche à la langouste.

20. Archives CEE. Direction générale des pêches. XIV/A/2. Rapport du 25 septembre 1987 du groupe " politique extérieure de la pêche ". L'article 354 de l'Acte d'adhésion prévoit que la communauté doit " préserver les activité de pêche " découlant des accords de pêche conclus avec un pays tiers.

21. Caseyeur : bateau spécialement conçu et équipé pour la pêche à l'aide de casiers (genre de masse utilisée pour la pêche des crustacés, art dormant).

22. Archives de la société brestoise FL Kuhn. Mauritanie 2. Texte de la déclaration de politique de développement du secteur de la pêche, p. 14. IV l'intégration des systèmes d'exploitation.

23. Archives de la société brestoise FL Kuhn. Mauritanie 2. Extrait d'un rapport confidentiel du programme de coopération FAO/Banque africaine de développement, RIM, développement de la pêche artisanale dans le sud et identification des possibilités d'investissement dans la filière langouste, rapport de préparation, 7 octobre 1987. Résumé et conclusion, p. III.

24. Ibidem. p. 31. 
plus qu'ils prévoient la fin rapide de l'activité bretonne : «Les unités de la flottille française sont toutes de vieilles unités (25 à 30 ans d'âge) et leurs armateurs ne veulent plus faire les investissements nécessaires pour les remplacer, puisque la poursuite de leur activité dépend entièrement de la politique du gouvernement mauritanien ${ }^{25}$."

Contrairement à l'avis de la FAO, en fin 1987, les armateurs bretons ne sentent plus l'activité menacée parce qu'elle est négociée dans le cadre communautaire. D'ailleurs, de nouveaux acteurs locaux investissent dans ce secteur : l'armement Dhellemmes de Concarneau rachète l'armement "France Langouste " de Douarnenez et la Société de mareyage Furic du Guilvinec devient majoritaire dans le langoustier camarétois Portzic en septembre 1987. Le rachat par l'armement Dhellemmes est porteur d'espoir à Douarnenez, les autorités maritimes n'hésitent pas à affirmer que " la continuité de l'activité langoustière est à présent assurée grâce d'une part à la conclusion d'un accord de pêche pluriannuelle entre la CEE et la Mauritanie, et d'autre part à la reprise par l'armement Dhellemmes de la société France Langouste ${ }^{26}$ ». À Douarnenez, nul ne peut envisager que l'armement industriel concarnois achète un armement moribond dans le but de récupérer les kilowatts des vieux bateaux, les projets de modernisation et de construction des langoustiers démontrent le contraire.

L'accord communautaire pluriannuel apporte la sécurité de la continuité de l'activité et également des subventions. Le renouvellement indispensable de la flottille ayant une moyenne d'âge de 27 ans en 1987, est envisagé pour l'année 1988 dans le cadre du Plan d'Orientation Pluriannuel (POP) 1988-1992 ${ }^{27}$. Ainsi, l'Europe Bleue porte l'espoir d'une modernisation de la flottille ${ }^{28}$. L'armement douarneniste prévoit une modernisation du Rio del Oro, du Notre-dame-de-Rocamadour et de l'Iroise à hauteur de 6 millions de francs par bateau. À Camaret, les projets de modernisation sont identiques pour le Saint-Rioc (refonte de la passerelle, révision du vivier, remotorisation, achat d'un enrouleur de chalut et installation d'un distillateur d'eau douce ${ }^{29}$ ). Cette modernisation est envisageable grâce à la durée de l'accord, à la réduction du coût des licences, aux aides nationales, régionales et départementales ${ }^{30}$. Par contre le Fonds Européen d'Orientation et de Garantie Agricole (FEOGA) n'accorde pas d'aide pour la moderni-

25. Ibidem, p. 31.

26. Archives des Affaires Maritime du quartier de Camaret. Monographie 1987, quartiers Douarnenez Camaret.

27. Programme d'Orientation Pluriannuel ou POP : instrument de gestion des pêches, institué dans le règlement CEE n 4028/86, il vise à adapter la capacité de capture à l'état de l'écosystème et exprime la puissance des flottilles en kilowatts.

28. SoRAIN, Domminique, "La modernisation de la pêche et son adaptation à l'Europe bleue ", Regards sur l'actualité, n 228 , février 1997.

29. Archives de la société brestoise FL Kuhn. Prod ar Mar 2.

30. Aide de l'AREDIPEB (association régionale pour le développement des industries de la pêche en Bretagne). Cette association se charge de verser des avances-relais sans intérêt aux armements qui bénéficient d'aide du FEOGA pour la construction ou la modernisation de bateaux de pêche hauturière. L'association est née le 19 décembre 1983. 
sation de bateaux de plus de 25 ans ${ }^{31}$. Pour cette raison, il est préférable de construire de nouvelles unités. La société FL Khun (armateur des trois grands langoustiers camarétois) signe le 27 novembre 1987 un protocole d'accord pour la construction d'un langoustier en fer de 34,4 m avec les établissements Piriou de Concarneau ${ }^{32}$, et bénéficie d'une aide de l'État et du FEOGA ${ }^{33}$, le reste du financement étant assuré par une société de quirats ${ }^{34}$ créée pour la construction du navire et d'un emprunt ${ }^{35}$. En remplacement des plus vielles unités (Joliot-Curie et du Claire-Jeanne), l'armement "France-Langouste" commande, en avril 1988, deux bateaux de 32,8 m³. La construction de ces deux langoustiers est envisagée dans le cadre d'une société mixte franco-mauritanienne. Selon P.-Y. Dhellemmes, la Mauritanie prévoit la constitution d'un armement national en remplacement de la flotte étrangère, il est donc impératif pour " France Langouste " de proposer sa technique et son savoir-faire pour éviter l'éviction de la zone de pêche. Cette proposition montre que les armateurs bretons évoluent sur la constitution d'une société mixte, ce projet ne les effraie plus comme ce fut le cas au début des années quatre-vingt, au contraire, elle leur assure la pérennité de la flotte langoustière ${ }^{37}$, elle devient le dernier recours de sa survie.

Dans le cadre de la CEE, avec des projets de renouvellement de la flottille, l'avenir de la pêche langoustière apparaît florissant en 1987. Pourtant c'est à partir de cette date qu'est perceptible la mort de la pêche langoustière finistérienne en Mauritanie.

\section{La désillusion du partage avec d'autres Européens}

"Nous sommes amenés à penser, pour notre métier, qu'il aurait été bien préférable d'en rester à la négociation directe des licences auprès du gouvernement islamique de Mauritanie quitte à les payer au tarif précédent de 350 US $\$$ le tjb, en gardant pour les Bretons l'exclusivité de cette pêche, plutôt que de payer des droits réduits à 121 écu le tjb au terme du nouvel accord établi par la CEE mais qui nous impose, sur zone la présence continue de huit navires portugais et auxquels il convient d'ajouter sou-

31. Archives de la société brestoise FL Kuhn. Mauritanie 2. Cahier de notes manuscrites de L. Kuhn. Note du 24 décembre 1987.

32. Archives de la société brestoise FL Kuhn. Camaret 8. Dossier prévision pour un nouveau langoustier 1987.

33. Archives de la société brestoise FL Kuhn. Camaret 8. Dossier prévision pour un nouveau langoustier 1987. Courrier du ministère délégué chargé de la mer à L. Kuhn le 15 février 1990 : l'aide n'ayant pas été utilisée, les crédits sont réaffectés sur d'autres projets.

34. Quirat : parts de copropriété d'un navire, le quirataire possède un ou plusieurs quirats.

35. Archives de la société brestoise FL Kuhn. Mauritanie 2. Dossier réalisé par L. Kuhn pour le ministère de la mer le $1^{\mathrm{er}}$ mars 1988.

36. Archives de la société brestoise FL Kuhn. Camaret 8. Dossier « France Langouste 1987-1988 "+ dossier intitulé FRAMAPECHE dans Mauritanie 2.

37. Archives de la société brestoise FL Kuhn. Mauritanie 2. Lettre de Pierre-Yves Dhellemmes au secrétariat d'État à la mer le 13 avril 1988. 
vent deux ou trois autres navires portugais affrétés par des Mauritaniens privés.

Les pertes d'exploitation, les pertes de matériel se chiffrent, aujourd'hui, à trois ou quatre fois la différence de coût des licences par navire et, nous craignons fort que si bon ordre n'est pas rapidement mis dans ce secteur les pertes d'exploitation imposeront un désarmement des navires.

Il est indispensable, Monsieur Le ministre, de persuader la Commission de Bruxelles que d'accorder les droits de pêche en Mauritanie aux Portugais, en compensation de l'accès à d'autres pêcheries, a été une grosse erreur ${ }^{38}$. "

Dès septembre 1987, la flottille langoustière portugaise devient supérieure à la flottille française ${ }^{39}$ en unité et en tonnage de navire : les licences autorisent l'activité de 10 unités françaises et de 8 unités portugaises (à cela s'ajoutent trois bateaux portugais sous licence privée et trois autres sous licence CEE/pélagique ${ }^{40}$ ). Les Français ont perdu l'exclusivité et le monopole de la pêche langoustière dans les eaux mauritaniennes.

Les Français regrettent le nombre de navires portugais et critiquent leurs techniques de pêche. Les Portugais pêchent au filet maillant. Les voyages en Mauritanie durent environ 35 jours, chaque bateau effectue 8 à 10 campagnes par an ramenant à chaque fois 4 à 5 tonnes de langoustes vivantes et 1 à 2 tonnes de langoustes congelées. L'équipage portugais se compose de 12 à 13 hommes travaillant en moyenne 12 à 13 heures par jour. Chaque navire embarque 400 filets de $60 \mathrm{~m}, 250$ filets sont mis en pêche et 150 en réserve représentant un total de $24 \mathrm{~km}$ dont 18 immergés. Au total, le tonnage global pêché estimé atteint 900 tonnes par $\mathrm{an}^{41}$. D'après un pêcheur portugais pêchant à l'hameçon sur les mêmes zones et qui se plaint des dégâts causés par les filets maillants ${ }^{42}$, les pêcheurs langoustiers portugais mouillent des filets dans lesquels viennent se prendre des poissons de toute sorte y compris les nobles et ils les laissent pourrir pendant quelques jours pour que cela serve d'appât aux langoustes.

« Les langoustiers portugais travaillaient avant dans le nord $22^{\circ}$ (Sahara espagnol) et comme ils ont dévasté cette partie en pêchant au filet droit (environ 30 filets de 300 mètres en nylon très bon marché) ils sont maintenant venus en Mauritanie où ils pratiquent ce même mode de pêche. Non seulement ils perdent des morceaux de filets dans lesquels, lorsqu'on les récupère, il y a des coques de langoustes, mais en plus lorsqu'ils accrochent aux roches ils lâchent les filets proprement dits ("la toile " coûtant très bon marché) et ils récupèrent les flotteurs et les plombs et mettent une autre toile. Mais ces

38. Archives de la société brestoise FL Kuhn. Mauritanie 2. Lettre de P.-Y. Dhellemmes à L. Le Pensec, ministre de la mer le 24 mai 1988.

39. Archives CEE. Direction générale des pêches. Background note sur l'Accord CEE/ Mauritanie, pêche à la langouste.

40. Archives CEE. Direction générale de la pêche. Note sur le « litige franco portugais pour la pêche à la langouste en Mauritanie ». Réalisée au printemps 1989 à la suite de la mission effectuée au Portugal par L. Kuhn, P.-Y. Dhellemmes et G. Oulhen.

41. Ibidem.

42. Archives de la société brestoise FL Kuhn. Camaret 6. Information extraite d'une note documentaire sur le Portugal. 
toiles prises dans le fonds ainsi que les morceaux de filets perdus restent pêchant, les langoustes se prennent dedans, se cassent les pattes et les antennes, elles meurent, pourrissent et les autres langoustes fuient. Avec les filets droits les pêcheurs prennent toutes les tailles de langoustes, particulièrement les grosses langoustes reproductrices qui, lorsqu'on les pêche au casier, ne viennent pas dans le casier car ne changeant pas de coque elles n'ont pas besoin de beaucoup de nourriture et l'appât qu'il y a dans les casiers ne les tente pas. Quand par hasard une grosse langouste reproductrice est quand même pêchée au casier nos marins la remettent à l'eau ${ }^{43}$. "

Dès 1987, la concurrence sur la zone de pêche se développe entre les deux flottilles, l'entente est au départ cordiale mais, très vite, face à la " mal pêche " des Portugais (décrite dans le témoignage précédent), les Bretons se montrent moins compréhensifs. "Aujourd'hui c'est la bagarre pour survivre et les affrontements se multiplient au risque de dégénérer dans des proportions alarmantes ${ }^{44}$ ", " Si au départ nous avons d'un commun accord joué la carte de la cohabitation passive, moyennant la perte ou la détérioration d'une bonne partie de notre matériel, ceci est désormais devenu impossible ${ }^{45} "$ : les tensions avec les Portugais s'intensifient au fil des mois. D'après le témoignage des patrons, L. Kuhn décrit les agissements des Portugais :

" Au lieu de faire des recherches, ils [les Portugais] sont venus directement sur les lieux de pêche des bateaux français que ceux-ci avaient mis de nombreuses années à trouver. Ils ont commencé dans le sud entre le $18^{\circ} 4$ et le $18^{\circ} 30$ et après avoir épuisé les ressources, sont venus dans le nord entre le $20^{\circ} 04$ et le $19^{\circ} 50$ où ils ont posé leurs filets.

Ils viennent donc sur les lieux de pêche traditionnels des bateaux français où ils trouvent nos bouées attachées aux filières des casiers. En l'absence des Français, qui eux relèvent les filières tous les deux à trois jours (puisqu'il faut entre temps qu'ils aillent de l'une à l'autre des filières, et pêchent l'appât) les Portugais relèvent leurs filets et en même temps tous nos casiers. Ils volent les langoustes qui s'y trouvent, souvent aussi prennent nos casiers.

Dans ces conditions, les bateaux français ne pouvant plus pêcher, ont bloqué les navires portugais et il y a eu un conflit ces tout derniers temps ${ }^{46}$."

Face aux agissements des Portugais, les pêcheurs bretons répliquent en coupant les filets portugais quand ceux-ci sont en travers de leurs filières de casiers ${ }^{47}$ : " Nous avons décidé de ramasser les filières encore existantes

43. Archives de la société brestoise FL Kuhn. Mauritanie 1. Compte rendu de la réunion des patrons langoustiers, le 10 avril 1987.

44. Archives de la société brestoise FL Kuhn. Mauritanie 2. Lettre de P.-Y. Dhellemmes à L. Le Pensec, Ministre de la Mer le 24 mai 1988.

45. Archives de la société brestoise FL Kuhn. Mauritanie 2. Extrait du rapport de Louis Spinec patron du Claire-Jeanne.

46. Archives de la société brestoise FL Kuhn. Mauritanie1. Lettre de L. Kuhn à Soumare Gaye, le 6 mai 1988 Annexe pratiquement identique jointe par L. Kuhn à un courrier adressé à la direction des pêches pour préparer la commission mixte de Bruxelles les 13 et 14 juin 1988.

47. Archives de la société brestoise FL Kuhn. Camaret 6. Dossier Portugal. Rapport d'un voyage effectué par Madec à Peniche au Portugal dans le but de se renseigner sur les pratiques des langoustiers portugais. 
et d'adopter une attitude plus hostile envers les navires portugais, ceci après avoir déposé plainte auprès des différentes autorités compétentes, mauritaniennes, françaises ainsi qu'à la $\mathrm{CEE}^{48}$. " Et effectivement en avril et mai 1988, les télex se multiplient pour informer de nouveaux incidents entre Bretons et Portugais : "Ces incidents répétés suscitent l'irritation croissante des autorités mauritaniennes qui envisagent la consignation des langoustiers français et portugais jusqu'à ce qu'ils concluent un accord à l'amiable ${ }^{49}$. "Mais à l'automne 1988, la crise s'intensifie "des armes à feu ont été utilisées ${ }^{50}$ ":

"Le langoustier camarétois Portzic, commandé par Jean Péron, a voulu remonter 150 casiers recouverts par des filets maillants portugais [...]. Déchirant les filets pour remonter sa pêche, le Portzic [...] a été pris en chasse par le Girassol [bateau portugais] [...]. Échouant dans sa tentative d'abordage, l'équipage a lancé des pétards à thon sur le navire français, puis tiré plusieurs coups de feu sans heureusement blesser personne. Le fileyeur portugais a été mis en fuite quand le Saint Rioc est venu au secours de son collègue $[\ldots]^{51}$. "

Pour les « Mauritaniens " bretons, les contraintes politiques rendent difficile leur vie de marins et aggravant leurs conditions de travail. Ils craignent pour leur activité et pour la préservation de la ressource. À partir du $1^{\text {er }}$ juillet 1988 , les filets maillants sont interdits pour la langouste, ils sont autorisés, comme les chaluts, pour pêcher l'appât mais uniquement jusqu'au 31 octobre 1989, le casier est le seul engin de pêche autorisé pour capturer des langoustes.

\section{Conséquence de l'accord : l'épuisement des stocks}

À partir de 1987, le nombre de bateaux exploitant le stock de langoustes roses passe de 10-11 à 23-24, aux bateaux français et portugais sous-licence, il convient d'ajouter les bateaux d'un armateur mauritanien Mohamed Salek ${ }^{52}$, " des bateaux pirates travaillant au Maroc ${ }^{53}$ " et ceux pratiquant la " fausse pêche ${ }^{54}$ ". Consécutivement, les captures augmentent

48. Archives de la société brestoise FL Kuhn. Mauritanie 2. Extrait du rapport de Louis Spinec patron du Claire-Jeanne.

49. Archives de la société brestoise FL Kuhn. Camaret 3. Télex du 28 avril 1988, télégramme du 3 mai 1988, Télex du 21 mai 1988.

50. Archives CEE. Direction générale de la pêche. Note «briefing " sur les relations de pêche avec la Mauritanie.

51. Archives CEE. Direction générale de la pêche. Copie d'un article de presse du 7 octobre 1988 de P. Attard.

52. Archives du Comité National Pêches Maritimes (CNPM). Comité Interprofessionnel de la langouste rose et verte, dossier 1973-1992, Compte rendu de la réunion du 6 janvier 1988 au Faou.

53. Archives CEE. Direction générale de la pêche. Note sur le « litige franco portugais pour la pêche à la langouste en Mauritanie ». Réalisée au printemps 1989 à la suite de la mission effectuée au Portugal par L. Kuhn, P.-Y. Dhellemmes et G. Oulhen.

54. Expression utilisée par les spécialistes notamment au CNROP. La fausse-pêche consiste à prélever un produit alors que le navire ne dispose pas de licence l'autorisant à le faire. Il s'agit donc d'une pêche clandestine. 
de façon significative : aux 800 tonnes des Français s'ajoutent les 900 tonnes des Portugais et la production nationale mauritanienne. De la situation d'équilibre maintenue depuis le début des années soixante-dix, on passe à une situation de sur-pêche ${ }^{55}$. Les capacités de pêches du stock, estimées à 800 tonnes selon le biologiste J. Maigret, sont largement dépassées. Et dès 1988 , les captures baissent sensiblement alors que le nombre de jours de pêche augmente de plus de $36 \%$ par rapport à 1987. Les prises par unité d'effort (PUE) ${ }^{56}$ sont réduites d'environ $50 \%$. Au printemps 1989, les pêcheurs portugais mentionnent une diminution des rendements de l'ordre de $20 \%$, ils font le plein de leurs bateaux en une vingtaine de jours au lieu de 8 à 10 jours en $1987^{57}$. Selon les armateurs français, la baisse de rendement de la pêcherie est évidente depuis l'arrivée des Portugais ${ }^{58}: 882$ tonnes en 1986-1987, 638 tonnes en 1987-1988 et 346 tonnes en 1988-1989. Le rendement moyen est passé de 400 à 200 puis à $60 \mathrm{~kg}$ par jour ${ }^{59}$. Pour compenser la chute des rendements, le prix de la langouste augmente ${ }^{60}$ : $34 \%$ au total. Cette " hausse anormale et malsaine " est due à un phénomène compensatoire artificiel qui ne permet cependant pas d'équilibrer les comptes d'exploitation ${ }^{61}$.

La raréfaction de la ressource halieutique provient de la surexploitation des fonds. L'État mauritanien a mis en place une politique lui permettant de récupérer des devises par la vente des licences mais pas une politique suffisamment protectionniste de la ressource en définissant des Total Allowable Catch (TAC) ${ }^{62}$ et en vérifiant le respect de l'interdiction de tel type d'engins de pêche. Les mesures prises n'ont pas réussi à préserver le stock dont la zone d'exploitation se réduit à une peau de chagrin. Victimes de la " sur-pêche " et de la " mal-pêche ", les richesses halieutiques s'épui-

55. Sur pêche ou overfishing : phénomène de surexploitation des ressources halieutiques qui a conduit à mobiliser des moyens de plus en plus perfectionnés, donc coûteux, pour accomplir les captures.

56. Prises : c'est la quantité totale de poissons, de crustacés et de céphalopodes capturée dans les eaux ou des zones de pêche données. On peut utiliser indifféremment les termes " capture" " produit " ou " prises ". Prises par unité d'effort (PUE) : quantité de capture par bateau.

57. Archives CEE. Direction générale de la pêche. Note sur le « litige franco portugais pour la pêche à la langouste en Mauritanie ». Printemps 1989.

58. Archives CEE. Direction générale de la pêche. XIV/A/2. Compte rendu de la réunion du 19 juillet 1989 sur le problème de la pêche à la langouste.

59. Archives de la société brestoise FL Kuhn. Mauritanie 2, note du 13 novembre 1989 chiffre concernant la pêche de l'"Équateur".

60. Archives de la société brestoise FL Kuhn. Camaret 6.

61. Archives CNPM. Dossier intitulé "Situation de la pêche langoustière en Mauritanie " 15 février 1989.

62. Total allowable Catch : Total admissible de captures ou quotas nationaux, mesure technique et politique de contrôle. CHAIGnEAU, Gaëlle, "L'Europe bleue et les marins pêcheurs artisans : impact d'une politique ou perversité des pouvoirs? (Exemples français et anglais)", dans : Pouvoirs et littoraux du $X v^{e}$ au $X x^{e}$ siècle, Acte du colloque international de Lorient (24, 25, 26 septembre 1998), LE BOUEDEC Gérard, CHAPPÉ François, dir., Rennes, PUR, 2000, 720 p., p. 558-559, explication des TACs. 
sent. La zone n'est plus rentable pour les langoustiers bretons. L'accord communautaire, tant attendu, a des conséquences catastrophiques pour la pêche langoustière française mais également pour la Mauritanie qui perd son stock de langoustes roses. Cette déception fait naître, chez certains Bretons, un sentiment anti-européen : "Laisser faire n'importe quoi par n'importe qui au mépris d'une ressource que l'on sait limitée, c'est prendre le risque de désorganiser l'existant. Cette politique du renoncement risque de nous être fatale. À force de vouloir être absolument européen, nous en oublions d'être Français. Les marins bretons n'accepteront pas d'être les cocus contents de l'Europe ${ }^{63}$."

\section{L'agonie de la pêche langoustière \\ Refuser l'inévitable par la lutte}

Dans la Bretagne littorale, les questions maritimes demeurent encore jusqu'aux années quatre-vingt-dix, un enjeu de taille pour les acteurs politiques $^{64}$. "La question est à présent devenue une affaire politique en France où l'intervention de la commission est de plus en plus sollicitée ${ }^{65}$. " Le litige de pêche franco-portugais se politise, relayé par des hommes politiques bretons au gouvernement (Louis Le Pensec, premier Ministre de la mer de 1981 à 1983; Ambroise Guellec, ministre de la mer de 1986 à 1988). À la fin de l'année 1988, les acteurs économiques et politiques multiplient leurs interventions et leurs actions. En octobre 1988, à Bruxelles, le ministre français de la mer évoque pendant 25 minutes le problème dans les eaux mauritaniennes, le « ministre portugais a été très ébranlé et s'est engagé à tout mettre en œuvre pour régulariser la façon de travailler de ses pêcheurs ${ }^{66}$ ". Fin novembre 1988, P.-Y. Dhellemmes dispose de trois minutes pour s'exprimer sur le problème franco-portugais devant le Président Mitterrand en visite à Concarneau ${ }^{67}$. Fin mars 1989, les armateurs L. Kuhn, P.-Y. Dhellemmes et le mareyeur G. Oulhen se rendent au Portugal pour trouver une solution en concertation avec les professionnels portugais pour " réduire impérativement l'effort de pêche " et préserver la ressource ${ }^{68}$. En avril 1989, P.-Y. Dhellemmes s'adresse par écrit au Premier ministre Rocard ${ }^{69}$. Les Chambres de Commerce de Quimper et de Brest, le président du Conseil

63. Archives de la société brestoise FL Kuhn. Camaret 8. Article d'Alain Le Bloas, «le coup de colère de l'armateur P.-Y. Dhellemmes », le 25 juin 1988.

64. Coulıou, Jean-René, La Pêche bretonne, les ports de Bretagne-sud face à leur avenir, PUR, Rennes 1997, p. 128 " un espace littoral choyé et objet de rivalité des politiques ".

65. Archives CEE. Direction générale de la pêche. XIV/A/2. note sur le "problème de la pêche à la langouste ", le 11 juillet 1989 .

66. Archive FLK. Mauritanie 2. Cahier de notes manuscrites de L. Kuhn. Note du 21 octobre 1988.

67. Télégramme du 28 novembre 1988.

68. Archives CEE. Direction générale de la pêche. Note sur le « litige franco portugais pour la pêche à la langouste en Mauritanie ". Printemps 1989.

69. Archives de la société brestoise FL Kuhn. Camaret 8. Dossier "France langouste " 1987-1988. 
régional de Bretagne, Y. Bourges, s'adressent à J. Mellick (ministre délégué auprès du ministre des Transport et de la Mer de juin 1988 à mai 1991) pour défendre les intérêts des armements bretons ${ }^{70}$. C. Miossec, président du Conseil général du Finistère, questionne le ministre délégué chargé de la mer sur le non-respect par le Portugal de l'accord signé le 14 mai 1987 entre la CEE et la République Islamique de Mauritanie ${ }^{71}$. Armateurs et politiques s'inquiètent du non-respect de l'accord par le Portugal mais également par la CEE qui n'applique pas ses engagements pris dans l'article 3 dans lequel elle « s'engageait à prendre toutes les mesures appropriées en vue d'assurer le respect par ses navires de l'accord et des réglementations régissant les activités de pêche de la Mauritanie ». En juin 1989, le député Cozan adresse une pétition à J. Delors, président de la commission européenne ${ }^{72} \ldots$

Pendant près de deux ans, les démarches et les recherches de solutions se multiplient à toutes les échelles comme l'illustrent l'abondance des archives de L. Kuhn, du Comité National des Pêches Maritimes et de la CEE. Mais les démarches n'aboutissent à aucun résultat. «Force est de constater que la pêche langoustière française n'a aucun poids politique ${ }^{73}$. " Effectivement, le poids de l'activité langoustière est essentiel pour Camaret et important pour Douarnenez mais ne pèse pas bien lourd dans la pêche française et encore moins dans la politique européenne des pêches.

\section{La mort}

Les bateaux de l'armement "France Langouste" se retirent de la pêcherie à la suite de la baisse des rendements de 50 \% en 1989. La société mixte envisagée par " France Langouste " n'a pas vu le jour, pas plus que le projet de construction des nouveaux bateaux. Pour tenter de maintenir le quota global de tonnage des navires attribué à la France, le Comité Interprofessionnel de la langouste aide le Groupement Langoustier en achetant des licences ${ }^{74}$ mais cela ne peut éviter le transfert des licences françaises, utilisées jusque-là par l'armement "France Langouste ", à la flottille portugaise : la France obtient des licences pour 6 navires et le Portugal pour 15 navires. À l'automne 1989, restent pour la flottille française les 5 langoustiers de Camaret et un petit langoustier, le Sainte Marine; les langoustiers douarnenistes ont désarmé au printemps. Mais à l'issue de la première

70. Archives CNPM. Comité Interprofessionnel de la langouste rose et verte. Lettre de L. Kuhn au Comité Interprofessionnel pour l'informer des multiples démarches réalisées.

71. Archives UAPF. Dossier Mauritanie. Intervention à l'Assemblée Nationale du 3 avril 1989.

72. Archives CEE. Commission des Communauté Européennes, Le Président. Courrier de J. Delors adressé à JY Cozan le 14 septembre 1989.

73. Archives CNPM, Comité Interprofessionnel de la Langouste rose et verte. Dossier 1973-1992. Compte rendu de réunion du 16 mars 1989 au Faou. Propos de P.-Y. Dhellemmes.

74. Archives du CNPM, Comité Interprofessionnel de la langouste rose et de la langouste verte, dossier 1973-1992. Compte rendu de la réunion du 25 juillet 1989 au Faou. 
campagne de 1989-1990, et bien que l'autorisation de pêcher « avec casiers et chalut pour l'appât du 10 février 1990 au 30 juin $1990^{75}$ " soit accordée, L. Kuhn décide de désarmer les trois grands langoustiers. Ils quittent définitivement la Mauritanie en janvier 1990 sans exploiter jusqu'au bout les licences qu'ils ont payées car les campagnes ne sont plus rentables.

L'accord réalisé en 1987 arrive à expiration en juillet 1990, de nouvelles négociations reprennent sur fond de querelle franco-portugaise et d'épuisement du stock de langouste. La CEE informe les armateurs qu'elle se refuse à demander une autorisation de pêche à l'appât au chalut et au filet maillant ${ }^{76}$. Pour sortir de l'impasse, les professionnels bretons demandent " l'arrêt de la pêcherie pendant une période de deux ans " pour permettre la reconstitution du stock et «l'octroi d'une prime d'immobilisation aux navires langoustiers ${ }^{77}$ ". Les armateurs appellent à l'aide l'État et la Communauté européenne pour subventionner une période d'arrêt de deux ans. Deux ans est une période courte pour la reconstitution des fonds mais cette période permet l'obtention d'une subvention. Ainsi les professionnels restent dans la perspective du maintien d'une pêcherie langoustière.

L'accord CEE-Mauritanie est reconduit du $1^{\mathrm{er}}$ août 1990 au 31 juillet $1993^{78}$, les langoustiers caseyeurs ont un droit de pêche pour 1950 tonneaux de jauge brute par mois en moyenne annuelle. Les Français demandaient non pas une limitation en tonnages mais en nombre de bateaux car les langoustiers portugais travaillant aux filets, ont de faibles tonnages mais une forte capacité de pêche ${ }^{79}$. Les casiers sont les seuls engins de pêche autorisés, la pêche à l'appât est interdite. Les redevances passent de 121 à 242 écus par tjb. Le tonnage des bateaux et la période de pêche sont limités mais ni la Mauritanie ni la CEE n'instaure de TAC ou de quotas ${ }^{80}$, or ce sont les seuls moyens vraiment efficaces de contrôler et donc de préserver la ressource. Face à cette lacune des textes, le syndicat des armateurs se déclare " indigné " devant les " orientations inconsidérées du Commissaire Marin ${ }^{81}$ ". Sans autorisation de pêche à l'appât, les Français ne demandent aucun tonnage.

75. Archives de la société brestoise FL Kuhn. Camaret 4. Courrier du MPEM à L. Kuhn le 22 août 1989.

76. Archives CEE. Direction générale de la pêche. Compte rendu de la réunion du 5 avril 1990 à Bruxelles.

77. Archives CEE. Direction générale de la pêche. Compte rendu de la réunion du 10 février/190 à Camaret, à laquelle participait un représentant de la CEE.

78. Archives UAPF. Dossier Mauritanie. Extrait du Journal Officiel des Communautés Européennes $\mathrm{N}^{\circ} \mathrm{L} 117 / 3$.

79. Archive FLK. Camaret 4. Lettre de L. Kuhn au ministère chargé de la mer le 31 mai 1990.

80. Archives UAPF, dossier Mauritanie. Document du secrétariat d'état à la mer, compterendu de la réunion de la commission mixte tenue dans le cadre de l'accord de pêche CEE/Mauritanie, à Bruxelles les 23-24 septembre 1991.

81. Archives UAPF, dossier Mauritanie. Courrier du président de l'UAPF au ministre délégué, le 12 décembre 1989. 


\section{La recherche des responsabilités}

Selon l'avis des scientifiques mauritaniens et du biologiste français J. Maigret, la pêche de la langouste au filet maillant a " certainement contribué pour beaucoup à l'effondrement des prises par unité d'effort, en effet ce type d'engin, non sélectif et destructeur pour le stock, capture de jeunes individus, et les morceaux de filets perdus continuent à mailler les langoustes qui pourrissent ${ }^{82}$ ". Cette pratique est plus dévastatrice que les chaluts des Bretons : utilisés seulement à certaines périodes et dans certains endroits, ils avaient préservé le stock de langoustes roses de 1970 à 1987. Dans ce but, les autorités mauritaniennes interdisent à partir du 31 octobre 1989 l'utilisation des filets maillants ${ }^{83}$.

Les pêcheurs français accusent également les filets maillants des Portugais, utilisés pour la langouste malgré l'interdiction. Les producteurs français accusent le "renoncement de l'administration française face à la $\mathrm{CEE}^{84}$ ", qui n'a pas défendu la pêche langoustière française en acceptant les langoustiers portugais en compensation de la perte de leur pêche démersale $^{85}$. Le ministre de la mer, J. Mellick, reproche à la CEE l'abandon de la pêche démersale portugaise ${ }^{86}$. Les producteurs rejettent également la responsabilité sur la Commission européenne pour n'avoir pas suffisamment contrôlé les navires portugais dans le cadre de l'accord ${ }^{87}$. Ils reprochent à la Mauritanie d'avoir, en dehors des accords communautaires, octroyé des licences privées et surtout de n'avoir pas agi avec fermeté face aux pratiques interdites des Portugais. Les marins ont porté plainte à plusieurs reprises auprès des autorités de Nouadhibou sans que cela soit suivi d'effets.

La Communauté européenne rejette les accusations des Français qui ont toujours été associés comme tous les États membres aux différentes négociations. La Commission rappelle qu'elle ne peut se substituer à l'autorité des États membres pour faire respecter l'accord par ses nationaux pas plus qu'elle ne peut se substituer à l'autorité des États tiers dans le contrôle de leurs eaux ${ }^{88}$. La Commission regrette aussi que les professionnels français et

82. Avis de Mika Diop, scientifique du CNROP, rencontré en mars 2003 à Nouadhibou.

83. Archives CEE. Direction générale de la pêche. Télex n 14363 du 8 novembre 1989 de la Commission Européenne à la direction générale des pêches à Lisbonne.

84. Archives de la société brestoise FL Kuhn. Mauritanie. Copie d'articles de presse sans source ni date qui cite P.-Y. Dhellemmes lors de l'assemblée générale de l'AREDIPEB.

85. Archives de la société brestoise FL Kuhn. Mauritanie 2. Compte rendu de la réunion pour la préparation des négociations entre la CEE et la Mauritanie, à Bruxelles le 5 avril 1990. Propos tenu par le représentant du ministère de la mer.

86. Archives de la société brestoise FL Kuhn. Mauritanie 2. Lettre de J. Mellick, ministre délégué chargé de la mer à M. Marin, vice-président de la Commission des Communautés européennes.

87. Archives CEE. Direction générale de la pêche. Bruxelles le 14 février 1990. Compte rendu de la réunion qui s'est tenue le 18 février 1990 à Camaret et à laquelle participait un représentant de la Commission.

88. Archives CEE. Direction générale de la pêche. Bruxelles le 14 février 1990. Compte rendu de la réunion qui s'est tenue le 18 février 1990 à Camaret et à laquelle participait un représentant de la Commission. 
portugais n'aient pas considéré l'alternative proposée par la Commission : l'abandon de la pêche à l'appât, tant au chalut qu'au filet maillant. Les professionnels préféraient le maintien d'un statu quo, voire l'utilisation pour tous du filet dans le but de maintenir les droits des Français, éventualité rejetée par la Commission qui refusait de « cautionner une politique à court terme qui aboutirait à la disparition rapide de la pêcherie langoustière, tant vis-àvis de ses propres pêcheurs que vis-à-vis des autorités mauritaniennes, elle avait une responsabilité quant à la conservation des ressources ${ }^{89}$ ". D'après ces propos, la CEE avait le sens des responsabilités, il lui manquait donc la capacité à faire respecter les règles. Les Portugais quant à eux accusent les Français d'avoir utilisé le chalut pour capturer les langoustes, et le considèrent aussi destructeur que le filet ${ }^{90}$.

De 1987 à 1990, trois éléments sont à l'origine de la destruction du stock : la surexploitation du fait de l'augmentation des capacités de pêche lors des accords CEE-Mauritanie, la "fausse-pêche " des flottilles étrangères et la "mal-pêche " due à la capture des langoustes avec des engins de pêche interdits. Quelle que soit la responsabilité de chacun, le résultat est sans appel : les fonds au large du Banc d'Arguin sont ravagés, le stock est détruit.

\section{Que faire de la flottille?}

«l'Europe Bleue doit prendre ses responsabilités et payer des dédommagements pour n'avoir pas su faire respecter les règlements ${ }^{91}$. " Les professionnels bretons demandent " le concours financier pour des projets de campagnes de pêche expérimentale à la langouste à Madagascar, en Namibie, aux Bahamas, au Brésil, au Costa Rica ou au Nicaragua ${ }^{92}$ ". Cette volonté d'une autre pêche langoustière ailleurs est menée de 1988 à 1991 par L. Kuhn. Il recherche des solutions pour ses grands langoustiers âgés mais bien entretenus car ils ne peuvent s'adapter à d'autres activités comme l'on fait les deux petits langoustiers pratiquant la pêche aux crabes. L. Kuhn prospecte et multiplie les projets cependant aucun n'aboutit ${ }^{93}$. Ces propositions renvoient aux prospections menées au cours des années soixante, vingt, trente ou cinquante dans les eaux d'Amérique Centrale, du Sud, d'Afrique du Sud et dans les mers australes. Aussi dans le cadre réglementé des accords communautaires et en ayant connaissance du droit de la mer, les armateurs espèrent

89. Archives CEE. Direction générale de la pêche. XIV/A/2. Bruxelles le 26 juillet 1989. Compte rendu de la réunion du 19 juillet 1989.

90. Archives CEE. Direction générale de la pêche. Compte rendu de la réunion du 5 avril 1990 à Bruxelles. Objectif de la réunion : pêche à la langouste en Mauritanie.

91. Propos extraits d'un article de J.-P. Louédoc dans Le Marin, du 9 février 1990, p. 11.

92. Archives CEE. Direction générale de la pêche. Compte rendu de la réunion du 10 février/190 à Camaret, à laquelle participait un représentant de la CEE.

93. Service Historique de la Marine à Brest. 11W397. Comité Interprofessionnel de la langouste rose et langouste verte. Compte rendu de la réunion du Comité Interprofessionnel du 6 mars 1992 à Brest. Propos de L. Kuhn. 
l'obtention de droits de pêche. Cependant la CEE finance la pêche expérimentale dans les eaux des États membres et des pays avec lesquels elle a conclu ou négocié un accord de pêche, et dans les eaux internationales ${ }^{94}$.

La CEE et le Maroc signent des accords de pêche le 23 juin $1988^{95}$. Ils autorisent pour une période de deux ans, une pêche expérimentale à la langouste aux casiers pour 1000 tjb dans la zone Sud à partir de 3 milles nautiques, contre une redevance de 33 écus par tjb. Fin 1988, selon les armateurs qui se sont rendus sur place ${ }^{96}$, sept bateaux portugais y pêchent au filet. C'est également au Maroc et vers la langouste verte que « France Langouste " cherche une alternative à la langouste rose. Rejetant la politique de licences, P.-Y. Dhellemmes envisage une société mixte, mode d'exploitation qui a été le serpent de mer de la pêche langoustière en Mauritanie pendant une dizaine d'années de 1978 à 1988. Il crée l'armement "Sud Langouste ", dont il détient $50 \%$ des parts, le reste appartenant à des capitaux marocains, ce qui suscite cette remarque : « À force d'être européens nous voilà contraints de devenir marocains ${ }^{97}$. " " France Langouste " assure la gestion technique mais le siège de l'armement est à Dakhla. Le Rio Del Oro, refondu aux Chantiers Piriou de Concarneau, est le premier bateau vendu à " Sud Langouste", suivi de l'Iroise. Les deux bateaux armés sous pavillon marocain pêchent la langouste verte au nord de Nouadhibou. La technique de pêche est celle des anciens verdiers ${ }^{98}$ de Douarnenez (pêche sur des annexes aux filets et conservation en vivier sur le bateau-mère), les bateaux sont commandés par d'anciens patrons verdiers, l'équipage est composé de douze hommes, six Douarnenistes anciens pêcheurs de langoustes vertes ou roses et six Marocains. La vente s'effectue à Douarnenez. La pêche, interdite d'octobre à février, réduit la période d'activité. Cette expérience marocaine avec les vieux outils de pêche, dure de 1989 à 1990, les conditions d'exploitation n'étant pas suffisamment rentables.

Le reste de la vieille flottille de "France Langouste " est désarmée : JoliotCurie désarmé depuis 1988 (détruit par la Marine Nationale, février 1989), Claire-Jeanne et Rocamadour en juin 1989. L'armement Kuhn, selon la volonté des quirataires, vend ses unités. La liquidation de la flottille langoustière s'inscrit dans le second POP ${ }^{99}$ (1987-1991). Les armateurs peu-

94. Archives CEE. Direction générale de la pêche. Compte rendu de la réunion du 10 février/190 à Camaret, à laquelle participait un représentant de la CEE.

95. Archives de la société brestoise FL Kuhn. Camaret 6 .

96. Archives de la société brestoise FL Kuhn. Mauritanie 2. Cahier de notes de L. Kuhn. Note du 19 janvier 1989 relatant le voyage de P.-Y. Dhellemmes au Maroc.

97. Expression de P.-Y. Dhellemmes reprise à plusieurs reprises dans la presse.

98. Verdiers : Nom donné aux pêcheurs de langoustes vertes. Les pêcheurs douarnenistes ont pêché la langouste verte dans les eaux du Rio de Oro (stock nord) et au Sud de Nouakchott (stock sud) de 1909 à 1969. Les marins embarquaient sur deux canots (appelés annexes) et pêchaient la langouste à la côte à l'aide de filets; ils ramenaient leur pêche dans le bateau-mère qui conservait la langouste vivante en vivier.

99. POP : plan d'orientation pluri-annuelle. Instrument de gestion des pêches, institué dans le règlement $\mathrm{CEE}{ }^{\circ}$ 4028/86, il vise à adapter la capacité de capture à l'état de l'écosystème et exprime la puissance des flottilles en kilowatts. 
vent obtenir une prime à la démolition accordée par la $\mathrm{CEE}^{100}$ à condition d'en avoir perçu une au niveau national. En 1991, le plan Mellick accorde des aides à la sortie de flotte pour l'arrêt définitif d'un navire ou son transfert définitif vers un pays tiers ou pour son affectation à d'autres fins que la pêche, à la condition de justifier d'au moins 100 jours d'activité en 1990. En dehors des primes à la démolition, les armateurs ont la possibilité de vendre les kilowatts des vieilles unités pour en construire de nouvelles. Les kilowatts des trois langoustiers douarnenistes désarmés ont permis la reconstruction en 1991 d'un chalutier de $26 \mathrm{~m}$. L'Armorique est vendu en février 1991 pour ses kilowatts ${ }^{101}$, sa société de quirats dissoute en juin, il est armé pour la plaisance. L'Équateur est vendu en janvier 1991 et sa société de quirats dissoute en juin; le bateau est transformé en fileyeur pêchant le thon l'été et les crustacés l'hiver, il s'échoue le 14 novembre 1994 au sud de Molène ${ }^{102}$. Le Saint-Rioc, vendu en janvier 1992, passe sous pavillon Saint-Vincent et Grenadines, la société de quirats est dissoute en juin ${ }^{103}$. Le Castel Dinn est vendu et transformé en crabier, le Portzic est désarmé ${ }^{104}$. Du fait de la structure financière des langoustiers camarétois (société de quirataires), leur liquidation a pris plus de temps.

Les kilowatts des bateaux vieillis servent aux armements essentiellement concarnois : le chalutier de «France Langouste " quitte Douarnenez pour Concarneau dès $1992^{105}$. L'armement Kuhn s'oriente également vers une autre pêche, le thon tropical, en partenariat avec les armements Chevannes-Merceron-Ballery ${ }^{106}$. Les bateaux désarmés, détruits ou vendus ne sont plus visibles dans les deux ports langoustiers.

En mars 1989, l'administrateur de Douarnenez et de Camaret affirmait que « la concentration d'une telle activité sur un territoire réduit augmente l'impact des difficultés qu'elle rencontre et rend plus difficile la gestion de la crise sociale qui en résulte ${ }^{107}$ ". Beaucoup d'anciens « Mauritaniens ", ayant atteint l'âge de la retraite avant 1989, quittent le métier sans rancœur. Quant

100. Archives de la société brestoise FL Kuhn. Mauritanie 2. Lettre de P.-Y. Dhellemmes à J. Mellick le 14 août 1989 .

101. Archives de la société brestoise FL Kuhn. Camaret 9. Contrat de cession de droit de pêche du langoustier " Armorique ".

102. Archives de la société brestoise FL Kuhn. Camaret 9. Extrait d'un article de presse de J.-L. Germain datant du 15/11/194 (pas de source).

103. Archives de la société brestoise FL Kuhn Mauritanie 2. Courrier de FLK le 10 mars 1993.

104. Archives du CNPM, Comité Interprofessionnel de la langouste rose et verte, dossier 1973-1992. Compte rendu de réunion du 6 mars 1992.

105. Idée développée par Jean-René Couliou : Couliou, Jean-René, LE BoulangER, JeanMichel, VILBROD, Alain, Port de pêche en crise. L'exemple de Douarnenez, L'Harmattan, 1998. p 25.

106. Service Historique de la Marine à Brest. 11W396. Langouste rose 1979-1989. Note du 11 octobre 1990.

107. Service Historique de la Marine à Brest. 11W397. Comité Interprofessionnel de la langouste rose et langouste verte. Commentaire de l'administrateur à la suite de la réunion du Comité Interprofessionnel du 16 mars 1989. 
aux équipages plus jeunes, ils se sont reconvertis à d'autres pêches : thon tropical (surtout pour l'état-major), chalut et pêche côtière. Leurs connaissances des nouveaux outils techniques leur permettent de s'adapter : les nouveaux patrons n'ont plus la nécessité de maîtriser les fonds de pêche dans la mesure où ils maîtrisent les techniques.

La mort de la pêche langoustière qui se solde par le désarmement des navires, est, à l'échelle de deux ports, une prémisse à ce que le géographe, spécialiste des pêches, J-R Couliou, appelle la mutation du système halieutique de Bretagne-Sud et qui débouche sur la " crise attendue " et la révolte des marins pêcheurs bretons en 1993-1994 ${ }^{108}$. Avec sa vieille flottille et ses équipages qui géraient le stock en " bons pères de famille " selon L. Kuhn ${ }^{109}$, la pêche langoustière bretonne n'a pas eu la capacité de résister aux concurrents européens : le conflit franco-portugais, a donné le coup de grâce à une activité déjà à bout de souffle. Dans une autre pêche, le conflit intra européen franco-espagnol du début des années quatre-vingt-dix met en difficulté la flottille hauturière française. Ces mutations résultent d'une volonté européenne, écologiquement louable, d'établir un équilibre entre l'effort de pêche et la ressource. Cet objectif est atteint dans le cadre de la pêche langoustière : effort de pêche nul pour une ressource en disparition. Malgré la mise en évidence de l'épuisement du stock, les principes de préservation de la ressource n'ont pas été respectés. Cela a provoqué chez les marins français et notamment bretons contraints d'abandonner leur flotte, l'impression d'être les laissés pour compte de la politique européenne de pêches. L'arrêt des flottilles langoustières incite la Mauritanie à demander des compensations à la CEE.

En perdant un chiffre d'affaire, des emplois, des bateaux, Camaret est anéanti et Douarnenez perd ce qui a longtemps été le fleuron de sa pêche. L'outil de travail des " seigneurs de la mer ", expression utilisée pour qualifiée les pêcheurs de langoustes, est vendu ou s'en va à la casse. Le départ et/ou la destruction physique des bateaux ont été vécus douloureusement par les anciens "Mauritaniens ": "Comme tous les matins, je pêchais dans la baie sur mon canot; j'ai vu le Claire-Jeanne partir, tiré par un remorqueur; il s'en allait sans fanfare au cimetière des bateaux à Landévennec; j'ai eu mal au cœur, ce bateau représentait 13 ans de ma vie ${ }^{110}$."

Comment dès lors faire le deuil de la pêche langoustière pour les acteurs et également pour les populations littorales marquées par la communauté des « Mauritaniens"?

108. Couliou, Jean-René, op. cit., Livre second.

109. Archives de la société brestoise FL Kuhn. Camaret 4. Lettre de L. Kuhn au ministère chargé de la mer le 31 mai 1990.

110. Témoignage de Jean Pencalet. 


\section{Faire le deuil de la pêche langoustière}

\section{À Douarnenez}

La pêche langoustière a été une pêche ostentatoire mais en marge de la vie du port. Moins de vingt ans après l'arrêt de l'activité, il en reste peu de traces physiques : les locaux de l'armement "France Langouste " dont le nom se voit de loin, quelques rares noms de rues ${ }^{111}$ et le Notre-Damede-Rocamadour, conservé dans la collection à flot du Port-Musée au Port Rhu. Dans ce dernier cas, l'outil de travail s'est transformé en objet du patrimoine, animé par des images vidéo, des photos et une bande-son.

Douarnenez a fait le choix d'une autre histoire, celle de la pêche sardinière "Douarnenez garde encore la réputation d'un grand port sardinier $[\ldots]^{112}$." Il ne reste pas plus de traces anciennes de cette activité, par contre des marques de construction récente rappellent le passé sardinier de Douarnenez : statue d'une sardine entre l'île Tristan et le Guet, panneaux touristiques jalonnant la ville sur le « chemin de la sardine ${ }^{113}$ ", exposition du Port Musée « l'art de fixer les saisons ${ }^{114}$ " rendant hommage aux femmes d'usines, filets de sardines accrochées aux fenêtres des particuliers durant la période estivale. Des films réalisés sur Douarnenez s'intéressent à la population sardinière : films de Marie Hélia L'Usine Rouge ${ }^{115}$ et Les Filles de la Sardine ${ }^{116}$ sur les femmes des usines; Les Caprices de la sardine de Patrick Le Ray présente un jeune patron pêcheur sardinier et à travers lui "l'histoire de cette communauté de marins et d'ouvrières de 1900 à nos jours, une histoire faite de joie et de misère mais aussi de conflits et de luttes liés à l'exercice d'un métier ${ }^{117}$ ". Dans ce documentaire la pêche langoustière apparaît cependant.

Douarnenez, ancien premier port sardinier de France, reste dans la mémoire collective la ville des " penn sardin " (nom choisi pour la nouvelle vedette de la société nationale de sauvetage en mer baptisée en juillet 2006). Cette activité marque l'identité de Douarnenez par sa durée, parce qu'elle a été l'activité dominante jusqu'à la fin des années cinquante, parce qu'elle touchait les marins et les femmes à l'usine en développant une " industrie de la sardine ${ }^{118}$ ", parce qu'elle signifiait la dureté au travail et des salaires

111. LAPPART Stéphane, Les noms de rues de Douarnenez, MM, UBO, 1990. Publié dans Mémoire de la ville, décembre 2007.

112. Extrait du site de la mairie de Douarnenez [ $w w w$. mairie-douarnenez.fr], rubrique " Découvrez Douarnenez ", partie " Douarnenez, toute une histoire".

113. Extrait du site de la mairie de Douarnenez [www.mairie-douarnenez.fr], rubrique " Découvrez Douarnenez », partie « Les chemins de la sardine ». Panneaux mis en place en 2005.

114. Inauguration de l'exposition du Port Musée au printemps 2006.

115. L'usine Rouge réalisé par Marie Hélia en 1989, récolte de témoignage de femmes âgées qui se souviennent de leur passé à l'usine.

116. Les filles de la Sardine réalisé par Marie Hélia en 2001, témoignage contemporain de femmes travaillant à l'usine à la fin du xxe siècle.

117. LE RAY Patrick, Les Caprices de la sardine, Film documentaire, Pois Chiche Film, 1996. 118. Le BOUlAnger, Jean-Michel, "L'industrie de la sardine, 1850-1990 », Mémoire de la ville, $\mathrm{n}^{\circ} 25$, Douarnenez, 1995. 
de misère pour les marins et les ouvrières, parce qu'elle a provoqué des luttes à terre ${ }^{119}$. Ce dernier élément illustre une autre image de Douarnenez, celle de " la ville rouge ". La pêche à la sardine donne à Douarnenez une image laborieuse et valeureuse conforme à une ville longtemps dirigée par un maire communiste. Or l'image véhiculée par la pêche à la langouste est tout autre. Les "Mauritaniens " deviennent selon l'expression du docteur Mével, les "Seigneurs de la mer ${ }^{120}$ ". Cette image contribue à faire de ces marins des hommes d'exception. L'appellation est donnée par la population qui ne fait pas partie de leur groupe comme l'écrit en 1964 le géographe F. Carré : "Ces pêcheurs appartiennent, en effet, à la vieille bourgeoisie de la pêche et forment un milieu traditionnel assez fermé qui justifie l'appellation de caste des "Seigneurs de la mer" souvent entendue au cours de conversation avec des Douarnenistes qui n'en faisaient pas partie. Les dynasties ont eu le temps d'apparaître : les Kernaléguen, Gloaguen, Pernès et Fiacre ${ }^{121}$. " Dans un même port, les groupes de pêcheurs se côtoient, mais sans se confondre. Progressivement, l'idée que les "Mauritaniens " constituent une " caste " parmi les marins s'impose surtout dans le regard des autres; les écrits des journalistes ont contribué à façonner cette image : René Pichavant du Télégramme, Philippe Attard Du Marin, Yves Allain, de Ouest-France. Ce dernier écrit : "Il n'est pas possible d'écrire l'histoire de Douarnenez sans faire référence à la pêche à la langouste ", la Verte, " qui a marqué l'histoire de Douarnenez et de ces fameux "Seigneurs de la mer" ". En 1981, le "Mois de la mer " met à l'honneur la pêche à la langouste rose. Cette valorisation des pêcheurs langoustiers perdure jusqu'à la fin de l'activité, comme l'illustre "Le chant du cygne de la langouste rose " article du Chasse-Marée écrit par le journaliste Laurent Giraudineau illustré par les photos de Michel Thersiquel (tous deux avaient embarqué sur le Notredame de Rocamadour pour son ultime campagne en 1989).

En s'émancipant du système sardinier douarneniste, le système langoustier est devenu autonome. Les marins se sont singularisés par leur absence. Chaque départ et retour des bateaux attiraient une foule importante sur les quais. De retour au port, les langoustiers se remarquaient par leur présence physique imposante, en baie ils tournaient pour "promener " leur cargaison de langoustes (indispensable pour éviter la mortalité de la cargaison), puis ils trônaient en majesté sur le slipway pour le débarquement. Les déchargements de langoustes se faisaient avec ostentation : le langoustier, hissé sur le slipway, vidé de son eau, se délestait panier par panier de ses milliers de langoustes. Le quai était alors animé par une centaine de personnes venues participer au débarquement, d'autres venaient en curieux,

119. LuCAS, Maurice, Luttes politiques et sociales à Douarnenez, 1890-1925, mairie de Douarnenez, 1975.

120. Mevel, Paul, Les Seigneurs de la mer, 1927, Saint-Brieuc, Éditions de la Bretagne touristique (réédition en 1998, Mémoire de La ville, Douarnenez).

121. CARRÉ, François, "La pêche lointaine de la langouste à Camaret et à Douarnenez ", Bulletin de la section de géographie, tome LXXVIII, année 1965, Paris Bibliothèque Nationale, 1966, pp 163-284, p. 223. 
pour glaner ou pour acheter une langouste faible. Après trois mois d'absence, certains "Mauritaniens " fêtaient leur retour dans les cafés du port. Certains marins se faisaient remarquer lorsqu'ils rentraient de campagne et certaines femmes affichaient des signes extérieurs de richesse ${ }^{122}$. Vue de la terre, la langouste porte en elle la notion de luxe et de richesse, aussi bien pour le produit que pour les producteurs.

Au cours des années quatre-vingt-dix, l'image change, l'image du "Seigneur de la mer " s'estompe avec la disparition de l'activité. On parlait dès lors de l'argent vite gagné, des fonds ravagés (tendance écologique aidant), à force de l'entendre dire, les marins, eux-mêmes, ont fait leur autocritique : "on a creusé notre trou ", " on a trop pêché ${ }^{123}$ ". Certains marins ont même eu tendance à dévaloriser la pêche à la langouste rose par rapport à celle de la verte : "Les vrais "Mauritaniens" étaient les verdiers, pas nous ${ }^{124}$. " La collection Ar Vag a réédité en fin 2005 le tome écrit sur les langoustiers en 1979, dans cette dernière édition, comme la précédente, la pêche à la verte est valorisée et celle de la rose rapidement présentée.

Contrairement à la pêche sardinière, la pêche langoustière n'a pas donné lieu à des luttes à terre. Quand il y en a eu, elles se sont menées en mer contre les rouleaux du Rio de Oro, contre les autorités mauritaniennes, contre les Portugais. Elle a laissé une image de pêche rémunératrice et destructrice. Les acteurs, pas plus que la population locale, n'ont perçu la marge de manœuvre très limitée des marins producteurs : pris dans les mailles d'un circuit économique, dépendants des enjeux politiques français, mauritaniens, portugais et européens ils n'avaient pas les clés pour freiner une évolution qu'ils ne maîtrisaient plus.

La mémoire collective se construit selon la volonté des groupes et au gré des volontés politiques. Actuellement Douarnenez valorise son passé sardinier. Le passé langoustier est-il encore trop proche, le souvenir de son échec trop récent, (et avec un échec en partie lié à l'Europe)? Le passage de la langouste nourricière à l'objet de culture maritime ${ }^{125}$ n'est pas encore complètement intégré. " Les traces issues de l'histoire et de la mémoire sont l'une et l'autre des constructions, toujours inachevées, en mouvement ${ }^{126}$ ", le travail de thèse sur les "Mauritaniens ${ }^{127}$ " participe à la construction de l'histoire langoustière. Quelles que soient les tendances mouvantes de la mémoire collective, de nombreuses mémoires individuelles sont marquées

122. Témoignage de Y. Kernaléguen. Là-bas si j’y suis, émission de D. Mermet sur France Inter, enregistrée le 21 octobre 1993.

123. Témoignage de Jean Pencalet, Yves et Théo Kernaléguen, Jean Gourmelen.

124. Remarque de Jean Pencalet.

125. Le Boulanger, Jean-Michel, PirIou, Nicole, " De la mer nourricière à la culture maritime, Douarnenez ", dans : PÉRON, Françoise, RieuCAU, Jean, La Maritimité aujourd'hui, Paris, L'Harmattan, 1996.

126. Couliou, Jean-René, Le Boulanger, Jean-Michel, Vilbrod, Alain, Port de pêche en crise. L'exemple de Douarnenez, L'Harmattan, 1998. p. 131.

127. Thèse de doctorat d'histoire soutenue par Françoise Pencalet-Kerivel à l'Université de Bretagne Sud en décembre 2006 sous la direction du professeur Gérard Le Bouëdec. 
par le passé langoustier de la ville parce qu'une partie importante de la population l'a vécu de près ou de loin. Sans avoir des revendications identitaires, donc sans risquer de dériver "vers l'apologie de soi et la haine de l'autre ${ }^{128}$ ", ces marins et leur famille ont eu un sentiment d'appartenance à un groupe particulier qui a existé. L'association " Mémoire de la ville " a publié un numéro consacré en grande partie à la pêche langoustière ${ }^{129}$, il a connu un succès public comme tous les numéros consacrés à la pêche ${ }^{130}$, confirmant l'intérêt et l'attachement de la population au passé maritime de la ville.

Selon le site Internet de la ville de Douarnenez, «Les Douarnenistes aiment leur réputation de turbulence et d'audace téméraire, qu'ils fondent sur leur histoire ${ }^{131}$ ". Mais ni le site de la ville ni celui de l'office de tourisme ne présentent quelques éléments de l'histoire langoustière ${ }^{132}$. À la différence du site de la ville de Camaret qui insiste sur son histoire maritime, celui de Douarnenez insiste surtout, non pas sur l'histoire, mais sur l'image laissée par son histoire que tout habitant anciennement ou nouvellement installé peut s'approprier.

\section{À Camaret}

« En 1990, le premier port langoustier d'Europe a tourné la dernière page d'une aventure commencée il y a près d'un siècle. Le long des quais, l'Équateur ou le Portzic ont attendu longtemps une nouvelle affectation. Dans les mémoires restent vivants l'audace et le savoir-faire de toutes ces générations de marins camarétois ${ }^{133}$."

À Camaret, l'arrêt de la pêche à la langouste rose en 1990 est l'ultime étape d'un long processus de déclin de la pêche langoustière. Celui-ci avait commencé en 1973 par l'arrêt de la pêche à la langouste rouge au Maroc à la suite de décisions politiques, puis, en 1979, les Camarétois avaient été contraints de quitter les eaux tunisiennes. Dès lors, reste à Camaret la pêche à la langouste rouge de l'Atlantique (en Bretagne et des îles Scilly à l'Irlande jusqu'aux Hébrides). Dans la monographie de $1982^{134}$, les autorités maritimes reconnaissaient un " ralentissement progressif et constant de l'activité du quartier de Camaret " n'hésitant pas à dire que le "vieillissement de la flottille et des marins" ne permettait pas de "reconquête possible de la mer par les Camarétois ". La pêche à la langouste rose est la dernière

128. Ibidem. p. 128

129. PenCAlEt-Kerivel, Françoise, " De la verte à la rose, voyage avec les Mauritaniens, un siècle d'aventure " dans Mémoires de la ville, $n^{\circ} 32,1999$, p. 7-94.

130. Propos de Jean-Michel Le Boulanger, responsable de l'association.

131. Extrait du site de la mairie de Douarnenez, www.mairie-douarnenez.fr, rubrique " Découvrez Douarnenez ", partie « Douarnenez, toute une histoire ".

132. [www.douarnenez-tourisme.com].

133. Extrait du site officiel de la mairie de Camaret sur Mer, [www.camaret-sur-mer.com], " nautisme, art et culture ", partie sur le patrimoine maritime.

134. Archives des Affaires Maritime du quartier de Camaret. Monographie de 1982. 
spécialité de Camaret et la dernière activité maritime importante : en 1986, elle représentait $25,66 \%$ des tonnages et $78 \%$ de la valeur des apports, mais en $1988,5,32 \%$ du tonnage et $53,56 \%$ de la valeur ${ }^{135}$. Aussi lorsque la crise langoustière se confirme à la fin des années quatre-vingts, les élus s'engagent pour tenter de préserver ce qui reste de l'activité langoustière. Sur leur initiative, une " commission des affaires européennes " est créée à la mairie de Camaret, elle a pour représentant E.L. Melenec, directeur de la "Marée Camarétoise " dernière entreprise de mareyage vivant de la vente des langoustes roses. Ce dernier organise une pétition (1080 signatures pour 3000 habitants) ${ }^{136}$ pour la préservation des droits de pêche en Mauritanie $^{137}$. Il s'adresse directement par lettre à J. Delors, président de la Commission européenne lui demandant d'intervenir pour faire respecter les accords : "Cette situation [...] ne peut, sans que la Commission n'y perde de sa crédibilité, perdurer ${ }^{138}$. " En 1989, la municipalité de Camaret commande une étude intitulée " La crise langoustière à Camaret sur mer : conséquences dramatiques sur l'économie locale ". Après une présentation de l'activité, l'étude alerte sur les conséquences économiques et sociales catastrophiques de l'arrêt de l'activité. Que va devenir la cinquantaine de marins inscrits à Camaret et quelle solution trouver pour les activités annexes qui vivaient jusqu'alors grâce à la langouste (chantier de construction, mareyage, avitaillement...)? Quel que soit le déploiement réalisé par la municipalité, le constat s'impose : aucune action ne peut éviter l'arrêt de la pêche langoustière. La mairie de Camaret et les armements des cinq navires entament une démarche judiciaire : représentés par un avocat au Conseil d'État et à la Cour de cassation, ils demandent à l'État la réparation du préjudice subi à la suite de la perte de l'activité tant par la commune que par les armements. Ils reprochent à l'État français de ne pas avoir agi pour que l'accord CEE-Mauritanie soit respecté ${ }^{139}$. Les Camarétois se sont battus pour la survie de la pêche à la langouste, ils jettent l'éponge devant l'inéluctable arrêt économique et entament une démarche culturelle et mémorielle.

En 1990, la mairie de Camaret envisage la création d'un Centre d'études de recherches et de formation sur la pêche langoustière et crustacés (Cerfoplanc) ${ }^{140}$. Pour l'aider dans sa démarche, elle s'adresse en juillet

135. Archives de la société brestoise FL Kuhn. Mauritanie 2. Dossier réalisé par Melle Melennec, " crise langoustière à Camaret sur Mer : conséquences dramatiques sur l 'économie locale " 1989 .

136. LE SCIELLOUR, "Camaret en mortes-eaux, I-La crise de la langouste sonne le glas de la belle époque ", le Télégramme, 13 mars 1990.

137. Archives de la société brestoise FL Kuhn. Mauritanie 2. Texte de la pétition datée du 18 juin 1989.

138. Archives de la société brestoise FL Kuhn. Mauritanie 2. Courrier de E L Melenec adressé J. Delors le 18 août 1989.

139. Archives de la société brestoise FL Kuhn. Camaret 9. Dossier de requête préalable réalisé par le cabinet d'avocat au conseil d'État et à la cour de cassation.

140. Archives de la société brestoise FL Kuhn. Camaret 6. PV de la réunion du conseil municipal de Camaret du 31 mars 1990. 
1990 au spécialiste de la langouste, Jacques Maigret, devenu conservateur de musée. Pour ce dernier, le Cerfoplanc à "vocation pluridisciplinaire et internationale " doit avoir des objectifs d'envergure : culturel (" faire revivre à Camaret l'époque de la langouste et redonner au port son identité culturelle $^{141}$ "), pédagogique, scientifique, technique et commercial ${ }^{142}$. Le projet très ambitieux et aussi coûteux ne voit jamais le jour. L'objectif culturel du projet de J. Maigret est repris et donne naissance à « La Maison du Patrimoine " qui collecte et conserve la mémoire langoustière de Camaret (objets, photographies, témoignages des acteurs...). Elle valorise le passé langoustier et l'expose dans un espace qui lui est consacré sur le port.

Au début du $\mathrm{XxI}^{\mathrm{e}}$ siècle, le site Internet de la municipalité de Camaret présente la nouvelle orientation de l'activité de la ville : "Après avoir connu une grande activité de pêche tout au long du $\mathrm{xx}^{\mathrm{e}}$ siècle avec son apogée dans les années soixante grâce à la pêche à la langouste, Camaret sur Mer est actuellement un port en mutation qui s'est doté d'une grande capacité d'accueil pour la plaisance et le tourisme ${ }^{143}$. "Camaret s'oriente nettement vers un développement touristique et considère son passé maritime comme un atout à valoriser. La pêche à la langouste dans son ensemble (rouge, verte et rose) fait partie de l'histoire de la ville, elle a valeur de patrimoine commun pour toute la population car elle était l'activité dominante. Son histoire est valorisée par des écrits, Les Années langoustes ${ }^{144}$, par des films, Rubis, au large du désert ${ }^{145}$ en 1962 et Les Mauritaniens de Camaret $^{146}$ en 1998, et, par la " Maison du Patrimoine ». Camaret a été le premier port langoustier européen et le revendique avec fierté.

\section{En Mauritanie}

En 1990, le stock de langouste verte est évalué à 220 tonnes (chiffre correspondant au potentiel de capture). Le stock nord, du Cap Blanc aux côtes du Sahara occidental, exploité jusqu'en 1975 par une flottille artisanale espagnole et quelques langoustiers franco-mauritaniens, est depuis exploité par une flottille artisanale mauritanienne à partir du port de La Guerra. La réduction de l'effort de pêche a favorisé la réapparition de la langouste verte dès 1981-1982 sur les fonds de pêche autour du Cap-Blanc et dans la baie du Lévrier d'où elle avait disparu depuis plusieurs décennies. La pêche sur le stock nord se pratique toute l'année contrairement

141. LOUEDOc, Jean-Paul, "La Mauritanie évanouie ", France Pêche, février 1991, p. 61.

142. Archives de la société brestoise FL Kuhn. Camaret 9. Courrier de J. Maigret adressé à la marie de Camaret le 22 août 1990.

143. Extrait du site officiel de la mairie de Camaret sur Mer, www.camaret-sur-mer.com, Port de plaisance.

144. Malbosc, Guy et Melennec, Roger, Les Années langoustes, Spézet, Keltia graphic Éditions, 1998, 110 p.

145. Fabiani, Henri, Rubis, au large du désert, court métrage noir et blanc, 13 minutes 13, 1962.

146. Zorninger, Alain, Les Mauritaniens de Camaret, moyen métrage de 25 minutes, 1998. 
au stock sud (au sud de Nouakchott) au caractère saisonnier (pêche au printemps et à l'automne). Ce deuxième stock est exploité artisanalement par des bateaux basés à Nouakchott et à Saint-Louis, une partie des captures est débarquée à Dakar. Sur des pirogues, les pêcheurs prélèvent 100 à 200 tonnes par an ${ }^{147}$ à l'aide de filets. Les pirogues ont remplacé les canots annexes des Douarnenistes. Conformément à la volonté du gouvernement mauritanien, cette pêcherie a été transférée des producteurs bretons aux producteurs mauritaniens mais indirectement du fait de la rupture dans le temps entre les verdiers douarnenistes et les pêcheurs mauritaniens.

Au début du Xxi ${ }^{\mathrm{e}}$ siècle, Mika Diop directeur de l'Institut Mauritanien de Recherche Océanographique et des Pêches (IMROP) évalue le stock de langoustes roses à 800 tonnes mais aucune flottille n'a de licence pour l'exploiter $^{148}$. Sans autorisation du chalut ou de filets maillants, les Portugais ont abandonné cette pêche après les Français. Les scientifiques de l'IMROP estiment qu'environ 200 tonnes sont prélevées en " fausse pêche " par des bateaux ayant des licences pour des pêches démersales. Ainsi malgré la volonté d'armateur mauritanien comme Ould Salek ${ }^{149}$, la Mauritanie n'a pas développé d'armement langoustier pour la langouste rose. Le transfert de technique de pêche des Bretons aux Mauritaniens ne s'est pas effectué, la société mixte, sujet de controverse pendant plus de quinze ans, était la clé nécessaire au transfert. Cette pêcherie fut pour les Mauritaniens comme pour les Bretons, l'histoire d'une occasion manquée.

Même si la flotte mauritanienne de pêche s'est accrue depuis les années quatre-vingt, la flottille étrangère est toujours importante (navires européens, russes, koweïtiens, japonais...). La situation des industries de pêche s'est améliorée grâce notamment à l'accroissement des quantités débarquées par la pêche artisanale ${ }^{150}$. Mais d'après un expert français travaillant à l'IMROP ${ }^{151}$, cela est insuffisant pour un réel développement du secteur de la pêche et pour un contrôle du prélèvement de la ressource. La Mauritanie dispose d'une immense richesse halieutique, mais par manque d'infrastructures à terre, de viviers et de stockage de congélation, l'essentiel de la production est toujours débarquée et vendue à l'étranger. L'accord signé le 20 juin 1996 entre l'Union Européenne et la Mauritanie offre des droits

147. Archives de l'Institut Mauritanien de Recherche Océanographique et des Pêches (IMROP). RIM, ministère des pêches et de l'économie maritime. Article de MIKA DIOP, "Les langoustiers ", Bulletin du CNROP, Volume 23, 1991, p. 198. 220 tonnes en 1987, $97 \mathrm{t}$ en 1988, 116 t en 1989. Groupe de travail sur les interactions technologiques en ZEE Mauritaniennes, CNROP, Nouadhibou, du 16 au 28 juin 1990, Archives ${ }^{\circ}$ 49, octobre 1990.

148. Rencontre de Mika Diop à l'IMROP à Cansado près de Nouadhibou, en mars 2003. 149. Mohamed Ould Salek, rencontré à Nouakchott en mars 2003, affrétait un langoustier dans les années quatre-vingt et espérait développer sa flottille. Il avait en sa possession les plans de pêche réalisé par les pêcheurs bretons.

150. Archives de l'IMROP. RIM, ministère des pêches et de l'économie maritime. Bulletin du CNROP Volume 23, 1991.

151. Philippe Tous : expert maritime, travaillait à l'IMROP au début du XxI ${ }^{\mathrm{e}}$ siècle. Rencontré en mars 2003 à Cansado, Nouadhibou. 
d'accès à la ressource mais aucune flottille ne l'utilise. Ainsi les défis actuels de la Mauritanie en matière de pêche sont la préservation de la ressource et sa gestion rationnelle ${ }^{152}$.

Cette ultime période de la pêche langoustière bretonne en Afrique occidentale est marquée par des bouleversements politiques. L'État mauritanien légifère et étend ses eaux territoriales espérant développer une pêche nationale et s'approprier son espace halieutique au détriment des armements étrangers ${ }^{153}$. Pour la pêche langoustière, les différents gouvernements mauritaniens n'ont pas établi de politiques visant à protéger la rente halieutique, sans doute car la flottille était limitée et la pratique ancienne. Les négociations communautaires, en autorisant une flottille plus importante, ont accru la pression sur les stocks. Évoquant l'aide au développement, la coopération, le partenariat, les institutions européennes légitiment les accords alors qu'ils constituent " un acte commercial d'acquisition de droits d'usage sur des ressources naturelles renouvelables ${ }^{154}$ „. De part et d'autres, France, Mauritanie, Europe, les acteurs politiques ont privilégié les intérêts économiques et non les intérêts écologiques de protection de la ressource. La perte de la flottille langoustière bretonne s'inscrit dans le cadre plus global des pêches bretonnes qui voient réduire ses unités à partir de 1988, processus renforcé lors de la crise de $1993{ }^{155}$.

Dans les deux ports bretons, qui s'étaient spécialisés dans la pêche langoustière en Mauritanie, cette activité fait partie du passé, sa fin a coïncidé avec l'accélération du déclin du secteur halieutique, aucune autre pêche n'a pris le relais. En fonction de leur vécu et de leur histoire propre, chacune des deux villes exploite ou pas l'image de ce passé langoustier.

152. Ould Hamady, Hob et Weigel, Jean-Yves, « La pêche en Mauritanie : une reconquête difficile ", dans : La Pêche en Mauritanie : enjeux et défis, Afrique Contemporaine, ${ }^{\circ}{ }^{187}$, juillet-septembre 1998, 143 p. p. 85.

153. LE CEUR, Christine, La Pêche en Mauritanie : du désert à la mer ou l'appropriation d'un espace halieutique, thèse de géographie, Montpellier 3, 1994.

154. Joseph CATANZANO, «Accords de pêche et régulation de l'accès Réflexions fondées sur les situations en Afrique de l'Ouest ", Communication du Séminaire international Les relations ACP/UE quelle voie vers des avantages réciproques? Secrétariat CTA/Commonwealth, 7-9 avril 2003, Secrétariat ACP, Bruxelles, Belgique.

155. Didou Henri (rapporteur), La mutation des pêches Bretonnes : un an après, Région Bretagne, $1^{\text {re }}$ réunion ordinaire, janvier 1995. 


\section{Bibliographie}

BEURIER, Jean-Pierre (dir.), Droits maritimes, tome III, Exploitation et protection de l'océan, Juris, Lyon, 1998, 310 p.

CADORET, Bernard, DuviaRD, Dominique, GuIlLEt, Jacques, KerISIT, Henri (dir.), Ar Vag, Voiles au travail en Bretagne Atlantique, Grenoble, éd. Les 4 seigneurs, 1979. Réédition 2005.

CARRÉ, François, "La pêche lointaine de la langouste à Camaret et à Douarnenez ", Bulletin de la section de géographie, tome LXXVIII, année 1965, Paris Bibliothèque Nationale, 1966, p. 163-284.

CATANZANO JOSEPH, "Accords de pêche et régulation de l'accès Réflexions fondées sur les situations en Afrique de l'Ouest ", Communication du Séminaire international Les relations ACP/UE quelle voie vers des avantages réciproques? Secrétariat CTA/ Commonwealth, 7-9 avril 2003, Secrétariat ACP, Bruxelles, Belgique.

Coulıou, Jean-René, La Pêche bretonne, les ports de Bretagne-sud face à leur avenir, Rennes, Presses universitaires de Rennes, 1997, 446 p.

CROIX Alain, LESPAGnOL André, (dir.), Les Bretons et la mer : images et histoire, Rennes, Apogée, Presses universitaires de Rennes, 2005, 192 p.

Didou, Henri (rapporteur), La mutation des pêches Bretonnes : un an après, Région Bretagne, $1^{\text {re }}$ réunion ordinaire, janvier 1995.

GIRAUDINEAU, Laurent, "Le chant du cygne de la langouste rose ", Le Chasse-Marée, $1990, n^{\circ} 48$, p. $2-15$.

KANE, Aïchétou, La Mauritanie et le droit de la mer, thèse de doctorat, droit, Université d'Orléans, 2003, 712 p.

Le BoulAnger, Jean-Michel, Douarnenez de 1800 à nos jours Essai de géographie historique sur l'identité d'une ville, Rennes, Presses universitaires de Rennes, 2000, $502 \mathrm{p}$.

Le Boulanger, Jean-Michel, PIRIOU, Nicole, « De la mer nourricière à la culture maritime, Douarnenez ", dans : PÉRON Françoise, RIEUCAU Jean, La Maritimité aujourd'hui, Paris, L'Harmattan, 1996, 335 p.

LE CEur, Christine, La Pêche en Mauritanie : du désert à la mer ou l'appropriation d'un espace halieutique, thèse de géographie, Montpellier 3, 1994.

MAIGRET Jacques, Contribution à l'étude des langoustes de la côte occidentale d'Afrique, (crustacea, decapoda, palinuridea) Panulirus regius brito Capello, 1864, Palinurus Mauritanicus Gruvel, 1911, Thèse de doctorat es sciences naturelles, Université d'Aix-Marseille, 1978, 264 p.

Ould HAMADY, Hob et Weigel, Jean-Yves, "La pêche en Mauritanie : une reconquête difficile ", dans : La Pêche en Mauritanie : enjeux et défis, Afrique Contemporaine, $\mathrm{n}^{\circ} 187$, juillet-septembre 1998, 143 p., p. 85.

PenCAlET-KeRIVel, Françoise, " De la Verte à la Rose, voyage avec les " Mauritaniens", un siècle d'aventures ", Mémoire de la ville [de Douarnenez], 1999, n 32, p. 794.

PenCALET-KeRIVEL, Françoise, Les « Mauritaniens » de 1945 à 1990, les pêcheurs langoustiers bretons dans un monde en mutation, Thèse de sciences humaines et sociales, discipline histoire, Lorient, 2006, 818 p.

Pencalet-Kerivel, Françoise, Histoire de la pêche langoustière, les "Mauritaniens " dans la tourmente du second $X X^{e}$ siècle, Rennes, PUR, 2008, 414 p. 


\section{RÉSUMÉ}

Douarnenez et Camaret se sont spécialisés au cours $\mathrm{du}_{\mathrm{Xx}} \mathrm{x}^{\mathrm{e}}$ siècle dans la pêche langoustière au large des côtes mauritaniennes. En 1987, la CEE se substitue aux États membres et négocie un accord avec la Mauritanie. Cet accord est porteur d'espoir tant en Bretagne qu'en Mauritanie car il semble assurer la continuité de l'activité. Cependant, il entraîne la perte de l'exclusivité bretonne : en tant qu'État membre de la CEE, les pêcheurs portugais ont accès aux eaux mauritaniennes, les deux flottilles européennes, françaises et portugaises, deviennent concurrentes. Différents acteurs locaux, nationaux et européens interviennent politiquement pour la survie de la flottille langoustière. Les tractations échouent, l'activité à bout de souffle meurt. En effet, la pression croissante sur un stock fragile provoque sa surexploitation et sa rapide destruction. En 1989-1990, la flottille langoustière disparaît du paysage portuaire, prémisses des mutations de la pêche bretonne. De façon différente, Douarnenez et Camaret font le deuil d'une activité qui a contribué à leur développement économique et qui a marqué les populations littorales.

\section{ABSTRACT}

In the course of the $20^{\text {th }}$ Century the cities of Douarnenez and Camaret specialised in the spiny lobster fishing industry along the Mauritanian coast. In 1987 the CEE centralised fishing agreements, instead of letting each member country make his own. It negotiated an agreement with the Mauritanian government that was at first considered full of promise by the Bretons and by the Mauritanians. However, it was to bring about the loss of Brittany's fishing exclusivity in the area, as any CEE member country could benefit from it. The Portuguese flotilla entered the area, and the two industries became competitors. Different local, national and European personalities tried to find political means to save the industry, but negotiations failed. The constant pressure of two flotillas on the fragile stocks of spiny lobster caused its depletion. The activity waned and became extinct; premise of the changes Brittany's fishing industry was to face the years to come. Douarnenez and Camaret reacted differently to the loss of this important economical and social factor. 